\title{
Frequency of cervical intraepithelial neoplasia following large loop excision of the transformation
}

\section{zone}

Geoffrey Hulman, Clive J Pickles, Clive A Gie, Fintan M Dowling, Philippa J Stocks, Reginald Dixon
Methods

We studied 669 consecutive patients aged between 17 and 75 years who had LLETZ biopsies showing CIN grades 1, 2, or 3 over a two year period. LLETZ with no CIN and microinvasive or invasive carcinomas were excluded from the study. Patients selected for LLETZ included not only those in whom CIN was clearly confined to the ectocervix but also those in whom the CIN extended into the endocervix.

The LLETZ biopsies were reported by three pathologists whose practice is to collaborate closely, particularly where difficulties are encountered with grading of CIN or the completeness of excision. Many of the more complicated LLETZ biopsies therefore had a consensus diagnosis

\section{GRADE OF CIN}

The LLETZ biopsies were subdivided according to the highest grade of CIN found in each case. The frequency of persistent/recurrent CIN was analysed for each grade of CIN in the LLETZ by a $\chi^{2}$ test for trend.

COMPLETENESS OF EXCISION OF CIN

The LLETZ biopsies were further categorised according to whether they showed complete, incomplete, or equivocal excision of CIN on histological examination. Equivocal excision was where completeness of excision could not be ascertained or where CIN extended "close" to an excision margin.

The frequency of CIN was compared by Fisher's exact test between those patients with complete and incomplete, complete and equivocal, and incomplete and equivocal excision of CIN. The patients had all been followed up with cervical cytology or histology, or both, over 1.5 to 3.5 years. The criterion for positive follow up was a histological diagnosis of CIN in samples such as cervical biopsy, repeat LLETZ, cold knife cone biopsy, and hysterectomy specimens. Follow up was considered negative if histology or cytology, or both, was normal.

The frequency of persistent/recurrent CIN was compared between patients with incomplete excision at the endocervical margin and those with incomplete excision at the ectocervical margin. The results were analysed by a $\chi^{2}$ test.

\section{Results}

Four hundred and fifty two LLETZ biopsies showed complete histological excision of CIN

\begin{abstract}
according to the histological grade and completeness of the original excision. the follow up results anticipated. A study was therefore carried out to assess the risk of persistent/recurrent CIN following LLETZ neered by Prendiville et al. ${ }^{1}$ It is now a wide cervical intraepithelial neoplasia (CIN). It has the advantage of being a potentially curativ specimen for histological diagnosis

The stimulus to carry out this study was an
\end{abstract} March 1998

\author{
Health Care Services
(NHS Trust),
Sutton in Ashfield,
Nottinghamshire, UK
Department of
Histopathology
G Hulman
F M Dowling
P J Stocks
Department of
Obstetrics and
Gynaecology
C J Pickles
C A Gie
R Dixon
Correspondence to:
Dr G Hulman, Department
of Histopathology, Kings
Mill Centre for Health Care
Services (NHS Trust),
Mansfield Road, Sutton in
Ashfield, Nottinghamshire
NG17 4JL, UK.
Accepted for publication \\ 5 March 1998 \\ Health Care Services
(NHS Trust),
Sutton in Ashfield,
Nottinghamshire, UK
Department of
Histopathology
G Hulman
F M Dowling
P J Stocks
Department of
Obstetrics and
Gynaecology
C J Pickles
C A Gie
R Dixon
Correspondence to:
Dr G Hulman, Department
of Histopathology, Kings
Mill Centre for Health Care
Services (NHS Trust),
Mansfield Road, Sutton in
Ashfield, Nottinghamshire
NG17 4JL, UK.
Accepted for publication \\ Health Care Services
(NHS Trust),
Sutton in Ashfield,
Nottinghamshire, UK
Department of
Histopathology
G Hulman
F M Dowling
P J Stocks
Department of
Obstetrics and
Gynaecology
C J Pickles
C A Gie
R Dixon
Correspondence to:
Dr G Hulman, Department
of Histopathology, Kings
Mill Centre for Health Care
Services (NHS Trust),
Mansfield Road, Sutton in
Ashfield, Nottinghamshire
NG17 4JL, UK.
Accepted for publication \\ Health Care Services
(NHS Trust),
Sutton in Ashfield,
Nottinghamshire, UK
Department of
Histopathology
G Hulman
F M Dowling
P J Stocks
Department of
Obstetrics and
Gynaecology
C J Pickles
C A Gie
R Dixon
Correspondence to:
Dr G Hulman, Department
of Histopathology, Kings
Mill Centre for Health Care
Services (NHS Trust),
Mansfield Road, Sutton in
Ashfield, Nottinghamshire
NG17 4JL, UK.
Accepted for publication \\ Health Care Services
(NHS Trust),
Sutton in Ashfield,
Nottinghamshire, UK
Department of
Histopathology
G Hulman
F M Dowling
P J Stocks
Department of
Obstetrics and
Gynaecology
C J Pickles
C A Gie
R Dixon
Correspondence to:
Dr G Hulman, Department
of Histopathology, Kings
Mill Centre for Health Care
Services (NHS Trust),
Mansfield Road, Sutton in
Ashfield, Nottinghamshire
NG17 4JL, UK.
Accepted for publication
}

(n) 
Table 1 Overall incidence of persistent/recurrent cervical intraepithelial neoplasia (CIN) following large loop excision of the transformation zone (LLETZ) biopsy of the cervix

\begin{tabular}{|c|c|c|c|c|}
\hline $\begin{array}{l}\text { Grade of CIN } \\
\text { in LLETZ }\end{array}$ & $\begin{array}{l}\text { Number of } \\
\text { LLETZ biopsies }\end{array}$ & $\begin{array}{l}\text { Incidence of persistent/ } \\
\text { recurrent CIN (\%) }\end{array}$ & $\begin{array}{l}\text { Grade of } \\
\text { persistent/recurrent CIN }\end{array}$ & Number (\%) \\
\hline \multirow[t]{4}{*}{3} & \multirow[t]{4}{*}{272} & \multirow[t]{4}{*}{$59(21.7 \%)$} & Adenocarcinoma in situ & $1(0.4 \%)$ \\
\hline & & & CIN3 & $15(5.5 \%)$ \\
\hline & & & CIN2 & $20(7.4 \%)$ \\
\hline & & & CIN1 & $23(8.5 \%)$ \\
\hline \multirow[t]{3}{*}{2} & \multirow[t]{3}{*}{277} & \multirow[t]{3}{*}{$37(13.4 \%)$} & CIN3 & $2(0.7 \%)$ \\
\hline & & & CIN2 & $13(4.7 \%)$ \\
\hline & & & CIN1 & $22(7.9 \%)$ \\
\hline \multirow[t]{3}{*}{1} & \multirow[t]{3}{*}{120} & \multirow[t]{3}{*}{$8(6.7 \%)$} & CIN3 & $1(0.8 \%)$ \\
\hline & & & CIN2 & $3(2.5 \%)$ \\
\hline & & & CIN1 & $4(3.3 \%)$ \\
\hline
\end{tabular}

Table 2 Incidence of persistent/recurrent cervical intraepithelial neoplasia (CIN) following complete, incomplete, and equivocal excision of CIN by large loop excision of the transformation zone (LLETZ) of the cervix

\begin{tabular}{|c|c|c|c|c|c|c|}
\hline \multirow[b]{2}{*}{$\begin{array}{l}\text { Grade } \\
\text { of CIN } \\
\text { in } \\
\text { LLETZ }\end{array}$} & \multicolumn{2}{|c|}{ Complete excision } & \multicolumn{2}{|c|}{ Incomplete excision } & \multicolumn{2}{|c|}{ Equivocal excision } \\
\hline & $\begin{array}{l}\text { Number of } \\
\text { LLETZ } \\
\text { biopsies }\end{array}$ & $\begin{array}{l}\text { Incidence of } \\
\text { persistent/ } \\
\text { recurrent } \\
\text { CIN }\end{array}$ & $\begin{array}{l}\text { Number of } \\
\text { LLETZ } \\
\text { biopsies }\end{array}$ & $\begin{array}{l}\text { Incidence of } \\
\text { persistent/ } \\
\text { recurrent CIN }\end{array}$ & $\begin{array}{l}\text { Number of } \\
\text { LLETZ } \\
\text { biopsies }\end{array}$ & $\begin{array}{l}\text { Incidence of } \\
\text { persistent/ } \\
\text { recurrent } \\
\text { CIN }\end{array}$ \\
\hline 3 & 143 & $15(10.5 \%)$ & 106 & $36(34.0 \%)$ & 23 & $8(34.8 \%)$ \\
\hline 2 & 200 & $19(9.5 \%)$ & 51 & $12(23.5 \%)$ & 26 & $6(23.1 \%)$ \\
\hline 1 & 109 & $4(3.7 \%)$ & 6 & $3(50.0 \%)$ & 5 & $1(20.8 \%)$ \\
\hline Totals & 452 & $38(8.4 \%)$ & 163 & $51(31.3 \%)$ & 54 & $15(27.8 \%)$ \\
\hline
\end{tabular}

$(67.6 \%), 163$ showed incomplete excision $(24.4 \%)$, and 54 showed equivocal excision $(8.0 \%)$. The frequency of persistent/recurrent CIN according to grade and completeness of histological excision in the LLETZ is give in tables 1 and 2 .

GRADE OF CIN IN LLETZ

The overall frequency of persistent/recurrent CIN strongly correlated with the grade of CIN in the LLETZ. The higher the original grade of CIN, the higher the frequency of CIN was found to be (table 1), irrespective of the excision status $(\mathrm{p}<0.0001)$.

COMPLETENESS OF EXCISION OF CIN BY LLETZ The frequency of CIN persistence/recurrence was significantly lower in patients with LLETZ showing complete excision of CIN $(8.4 \%)$ than in those with incomplete $(31.3 \%)(\mathrm{p}<0.0001)$ or equivocal excision $(27.8 \%)(\mathrm{p}<0.0001)$. However, despite a histological report of complete excision of high grade CIN (CIN 2 and 3), 34 of these 343 patients $(9.9 \%)$ developed recurrent CIN, 14 of whom $(4.1 \%)$ developed CIN 2-3.

Twelve of 62 patients in whom CIN was incompletely excised at the ectocervical margin and 32 of 77 patients with incomplete excision at the endocervical margin developed persistent/recurrent CIN. The frequency of subsequent CIN was significantly greater following incomplete excision at the endocervical margin than at the ectocervical margin $(\mathrm{p}<0.005)$.

There was no significant difference between the incidence of CIN following LLETZ with incomplete and equivocal excision of CIN ( $p=$ 0.7335 ). The group of patients who had the greatest risk of recurrent CIN were those with incompletely or equivocally excised CIN 3. Approximately one third of these developed CIN (table 2). The group of patients with completely excised CIN 1 was least likely to develop recurrent CIN.

One patient who had incompletely excised CIN 1 in the LLETZ biopsy had CIN 3 at follow up. Her cervical smear, however, showed severe dyskaryosis before the LLETZ, which did not reflect the severity of changes in the smear. One patient with CIN 3 in the LLETZ had adenocarcinoma in situ and CIN 1 at follow up. No patient had invasive carcinoma at follow up.

One patient had a follow up smear where the classification of tuboendometrial metaplasia or severe dyskaryosis was uncertain. No lesion was identified colposcopically. Subsequent cervical cytology was negative.

There were 22 patients with minor cytological abnormalities at follow up (mild dyskaryosis and borderline changes) but without positive biopsy confirmation. Nineteen of these patients had subsequently normal cervical cytology, colposcopy, or biopsy. No further follow up was available on three patients who had borderline smears following LLETZ.

\section{Discussion}

When performing an LLETZ biopsy, a balance has to be struck between preserving as much healthy cervix as possible and removing a sufficient margin of normal cervix to achieve complete excision of CIN. In addition, the full extent of CIN within the endocervical canal may be invisible to the colposcopist. Thus some LLETZ biopsies fail to excise CIN fully.

Incomplete excision was significantly more common with high grade CIN. This is not surprising as high grade CIN tends to be more extensive than low grade CIN-30.1\% of our patients with CIN 2 or 3 in their LLETZ biopsies had incompletely excised CIN. Felix et al reported similar results with incomplete excision in $33.3 \%$ of patients with CIN 2 or 3 in their LLETZ biopsies. ${ }^{2}$

Persistent or recurrent CIN is significantly less common following incomplete excision at the ectocervical margin than at the endocervical margin, perhaps because of destruction of residual CIN on the ectocervical aspect of the LLETZ bed by cauterisation.

Difficulties encountered identifying excision margins owing to diathermy artefact, multiple piece LLETZ (top hat), presence of skip lesions (as a result of previous cervical surgery), and the development of de novo CIN all contribute to discrepancies between the histological report of the margins and follow up findings. A histology report of complete excision of CIN should not therefore imply definite cure of CIN. Conversely incomplete excision of CIN should not imply definite residual CIN.

In addition, there is a variation between colposcopists in the criteria used in the selection of patients for LLETZ. This will result in a variation in the frequency of persistent/ recurrent CIN between colposcopists. Persistent/recurrent CIN may be expected in some patients, particularly when LLETZ is carried out primarily as a diagnostic procedure. 
It should therefore not be construed as treatment failure or evidence of poor practice.

The frequency of recurrent or persistent CIN was $31.3 \%$ in those patients with incompletely excised CIN on the original LLETZ. Bigrigg et al reported recurrent CIN in 33\% of LLETZ biopsies showing incompletely excised CIN. ${ }^{3}$ Felix et al reported residual CIN in $63 \%$ of patients who had re-excision of the endocervical area following incomplete LLETZ excision of high grade CIN. ${ }^{2}$ In contrast, Murdoch et al reported residual disease at three months following incomplete LLETZ excision of CIN in only 26 of 256 patients $(10.2 \%){ }^{4}$

There was a high frequency of persistent/ recurrent CIN in patients with equivocal excision of CIN (27.8\%). These figures suggest that such patients should be treated in the same way as those with unequivocally incomplete excision of CIN. It is interesting that a histology report of equivocal excision, which may initially appear confusing in attempts to plan appropriate patient management, in our experience has significant and useful prognostic value.

Careful follow up, including colposcopy, after incomplete LLETZ excision of CIN has been widely recommended. ${ }^{2-4}$ The risk of recurrent CIN was so great following not only incomplete but also equivocal excision of CIN that we feel colposcopic follow up for both groups of patients should be considered.

Although the number of patients with incomplete and equivocal excision of CIN 1 was very small, we feel even they should be followed up very carefully and preferably colposcopically, particularly if there is a discrepancy between grade of CIN in the LLETZ and degree of dyskaryosis in previous cervical smears. Incompletely or equivocally excised CIN 1 may occasionally represent the "tail end" of residual high grade disease.

A more difficult problem is the appropriate management of patients with completely ex- cised high grade disease. Our findings support those of Goff et al, that high grade CIN is also an important risk factor for recurrent CIN. ${ }^{5}$

Ten per cent of patients with completely excised high grade CIN developed recurrent CIN. Colposcopic surveillance for all these patients is clearly not feasible. It is, however, important they be followed up carefully with competently taken cervical smears, as $4 \%$ of this group developed high grade CIN during the 1.5 to 3.5 year follow up period. Adequate endocervical sampling is essential. Buntinx and Brouwers have shown that this may be achieved by either extended tip spatula, spatula and endocervical brushing, or cervix brush.

Currently, patients with completely excised CIN are followed up with annual smears for five years before being returned to normal three yearly recall if negative. We are now investigating the long term risk of recurrent CIN following complete excision of high grade CIN as a further refinement of appropriate follow up protocols.

We thank Dr M Blair and Jane Jenkins for assistance with statistics and Mrs L West and Mrs K Freeman for secretarial assistance.

1 Prendiville W, Cullimore J, Norman S. Large loop excision of the transformation zone (LLETZ). A new method of
management for women with cervical intraepithelial management for women with cervical intraepi

2 Felix JC, Muderspach LI, Duggan BD, et al. The significance of positive margins in loop electrosurgical cone biopsies. Obstet Gynecol 1994;84:996-1000.

3 Bigrigg A, Haffenden DK, Sheehan AL, et al. Efficacy and safety of large loop excision of the transformation zone. Lancet 1994;343:32-4.

4 Murdoch JB, Morgan PR, Lopes A, et al. Histological incomplete excision of CIN after large loop excision of the transformation zone (LLETZ) merits careful follow up, not retreatment. Br F Obstet Gynaecol 1992;99:990-3.

5 Goff BA, Rice LW, Fleischlhacker DS, et al. Large loop excision of the transformation zone in patients with exocervical squamous intraepithelial lesions. Eur $\mathcal{f}$ Gynaecol Oncol 1994;15:257-62

6 Buntinx F, Brouwers M. The relation between sampling device and detection of pathology in cervical smears. BMf 1996;313:1285-90. 